\title{
Vias para Nutrição Artificial na Criança (I): Acessos Entéricos
}

\author{
Artificial Nutrition in Children (I): Enteral Access
}



\author{
José ESTEVÃO-COSTA ${ }^{1}$ \\ Acta Med Port 2014 Sep-Oct;27(5):634-640
}

RESUMO

O aporte nutricional adequado é crucial na abordagem terapêutica de múltiplos estados mórbidos, o que justifica o recurso frequente e crescente a vias de acesso específicas para nutrição entérica e parentérica. No presente artigo é realizada uma revisão da literatura relevante acerca de indicações, modalidades, eficácia e complicações das vias de acesso entérico na criança. Os fatores a considerar na decisão entre acesso gástrico e acesso pós-pilórico, e entre colocação de sonda nasogástrica e gastrostomia são criteriosamente discutidos. Perante indicação para acesso gástrico de longa duração, a gastrostomia endoscópica percutânea é a técnica mais utilizada dada a sua eficácia e segurança, embora a morbilidade associada não seja desprezível; a colocação de gastrostomia laparoscópica emerge como uma alternativa potencialmente vantajosa.

Palavras-chave: Criança; Nutrição Entérica; Intubação Gastrointestinal; Gastrostomia; Jejunostomia; Laparoscopia.

\section{ABSTRACT}

Appropriate nutritional support is crucial in the therapeutic approach of multiple conditions, which justifies the frequent and increasing use of specific access routes for enteral and parenteral nutrition. This article reviews the relevant literature concerning indications, procedures, effectiveness and complications of enteral access routes in children. The decision between gastric and postpyloric access, and between nasogastric tube and gastrostomy is thoroughly discussed. Percutaneous endoscopic gastrostomy is the most commonly used technique when a long-term gastric access is required, given its efficacy and safety although the associated morbidity is not negligible; laparoscopic gastrostomy emerges as a potentially advantageous alternative.

Keywords: Child; Enteral Nutrition; Intubation, Gastrointestinal; Gastrostomy; Jejunostomy; Laparoscopy.

\section{INTRODUÇÃO}

O estado nutricional tem uma importância crucial no processo de recuperação e cura, sendo reconhecida a sua influência nas taxas de mortalidade e morbilidade associadas à doença principal. Na criança, o estado nutricional adquire ainda maior relevância pois, ao consumo basal e do stress dependente da doença ou traumatismo, acrescem as necessidades inerentes ao crescimento e maturação num ser com reservas muito limitadas.

O conhecimento mais aprofundado de múltiplos aspetos relacionados com a nutrição artificial, assim como alguns avanços tecnológicos, resultaram num aumento substancial da utilização de suporte nutricional artificial na criança e, consequentemente, do recurso a vias de acesso para nutrição entérica e parentérica.

A indicação para iniciar nutrição artificial deve ter caráter proativo. Um adulto, previamente saudável, poderá manter até sete dias um aporte nutricional artificial satisfatório, sem necessidade de vias de acesso específicas; na criança, este período tem menor duração, sendo aconseIhável que no lactente não ultrapasse quatro a cinco dias. ${ }^{1}$ Assim, devem prever-se as situações com risco de maior duração de impossibilidade de alimentação oral eficaz, antecipando a colocação de uma via de acesso para nutrição artificial.

O conceito atual de nutrição entérica abrange não só o aporte de nutrientes por sonda/tubo ou enterostomia, mas também o uso de nutrientes por via oral; no presente artigo apenas é contemplado o primeiro aspeto.

\section{INDICAÇÕES}

Perante indicação para nutrição artificial, se a função gastrointestinal for adequada, deverá eleger-se a via entérica dada a sua eficácia, simplicidade, segurança e efeito trófico sobre a mucosa intestinal. ${ }^{1} \mathrm{~A}$ estas vantagens da nutrição entérica relativamente à parentérica acresce o custo financeiro substancialmente mais baixo e a ausência de complicações graves tais como a sépsis relacionada com o cateter e a toxicidade hepática. ${ }^{2}$

A administração das soluções nutritivas diretamente no trato gastrointestinal pode estar indicada em doenças do tubo digestivo ou afeções extradigestivas. A obstrução, funcional ou orgânica, do mecanismo de deglutição é uma indicação comum para nutrição entérica (p.e. prematuridade $<32$ semanas, traumatismo crânio-encefálico, paralisia cerebral, estenose esofágica). Quando a tolerância entérica não permite a satisfação integral das necessidades nutricionais, deverá manter-se a administração entérica de pequenos volumes de nutrientes 'tróficos' com o objetivo de prevenir uma deterioração adicional da função intestinal. ${ }^{3,4} \mathrm{~A}$ nutrição entérica, inclusive com recurso a vias de acesso 'invasivas', tem registado uma aplicação crescente e benéfica em situações de hipercatabolismo (p.e. grandes queimaduras), na melhoria do estado nutricional (p.e. fibrose quística, cardiopatias, doenças metabólicas) e no inten-

1. Serviço de Cirurgia Pediátrica. Hospital S. João/Faculdade de Medicina. Universidade do Porto. Porto. Portugal.

Recebido: 02 de Março de 2014 - Aceite: 19 de Maio de 2014 | Copyright @ Ordem dos Médicos 2014 
sivismo em geral..$^{5-9}$

\section{TIPOS E TÉCNICAS DE ACESSOS}

\section{Acesso Gástrico versus Acesso Pós-pilórico}

A infusão no tubo digestivo pode ser efetuada no estômago ou no duodeno/jejuno (Tabela 1). A administração gástrica tem inúmeras vantagens relativamente à pós-pilórica, nomeadamente: maior simplicidade e segurança na colocação da via de acesso; digestão e resposta hormonal mais fisiológicas; efeito bactericida do ácido clorídrico; e, melhor tolerância a altas cargas osmóticas e maiores volumes com consequente menor incidência de síndrome de dumping. ${ }^{1,2}$

A infusão pós-pilórica apresenta a vantagem de minimizar o risco de aspiração traqueobrônquica e, em certas circunstâncias, permitir o início precoce da nutrição entérica. A sua utilização na criança é rara mas tem sido crescente, não se restringindo aos casos com alto risco de aspiração, sendo útil se a administração intragástrica não é tolerada (p.e. gastroparésia), se o acesso ao estômago não é viável (p.e. transposição gástrica), ou quando se pretende evitar a estimulação da secreção pancreática (p.e. pancreatite, pseudoquisto pancreático).

\section{Sonda Nasogástrica}

Existem sondas nasogástricas de diferentes composições: as de polivinil são as mais rígidas e por isso de colocação fácil, mas são pior toleradas e deverão ser substituídas a cada três a cinco dias. As de poliuretano e as de silicone são menos rígidas, de colocação mais difícil, mas por serem bem toleradas são as mais utilizadas em nutrição entérica prolongada, podendo permanecer até oito semanas sem necessidade de substituição. ${ }^{2}$

A colocação da sonda deverá efetuar-se pelo nariz aproveitando o reflexo da deglutição, calculando-se a extensão a introduzir como a distância do nariz à cicatriz umbilical. A verificação da correta localização após colocação é importante, dadas as consequências desastrosas de uma eventual administração traqueobrônquica. ${ }^{2}$ Caso não seja possível aspirar conteúdo gástrico (com eventual confirmação de um pH inferior a 3) deverá efetuar-se uma radiografia simples, pois a maioria das sondas são radiopacas. ${ }^{10,11}$

As complicações relacionadas com a intubação nasogástrica não são desprezíveis. A deslocação secundária da sonda nasogástrica é relativamente frequente e ocorre geralmente em sentido proximal, para o esófago. Neste caso, surgem vómitos, com alto risco de aspiração brônquica, após a administração. A perfuração da faringe é uma

Tabela 1 - Acessos entéricos

\section{A. Gástrico}

1. Sonda nasogástrica

2. Gastrostomia

2.1 Cirúrgica

- $\quad$ por laparotomia: clássicas; botão

- $\quad$ minimamente invasiva (laparoscópica)

2.2 Percutânea

- $\quad$ endoscópica (PEG)*

- $\quad$ radiológica

\section{B. Pós-pilórico}

1. Sonda naso (duodenal) jejunal: com ou sem controlo radiológico/endoscópico

2. Jejunostomia

2.1 Cirúrgica

- por laparotomia

- $\quad$ minimamente invasiva (assistida por laparoscopia)

2.2 Percutânea

- $\quad$ através de gastrostomia

- $\quad$ direta (endoscópica) 
complicação excecional, ocorrendo geralmente em prematuros de peso inferior a 1500 gramas, e pode induzir um falso diagnóstico de atrésia esofágica. A deslocação para jusante do piloro pode ocasionar diarreia osmótica e desregulação glicémica (síndrome de Moncrieff). A rinofaringite e a otite média são intercorrências frequentes, sobretudo com as sondas rígidas, e justificam a desinfeção rinofaríngea diária. A irritação da mucosa gástrica ou duodenal pode resultar em ulceração, estenose ou perfuração, mas são extremamente raras com sondas de consistência mole. O papel de uma sonda nasogástrica como precipitante de refluxo gastroesofágico (RGE) é controverso; por um lado, pode favorecê-lo por induzir esofagite microtraumática com consequente diminuição da depuração esofágica; por outro lado, a nutrição entérica pode proteger contra o RGE por diminuir a pressão intragástrica e a frequência dos relaxamentos transitórios do 'esfíncter esofágico inferior' relacionados com a deglutição. ${ }^{12,13}$

\section{Sonda Nasogástrica versus Gastrostomia}

Para além das complicações acima referidas, a intubação nasogástrica apresenta inconvenientes que limitam o seu uso, sobretudo quando prolongado. A estimulação das secreções orofaríngeas, a aerofagia e a dificuldade respiratória adicional que a sonda representa num recém-nascido podem constituir inconvenientes significativos. O 'desconforto' rinofaríngeo e a 'agressão' psicológica por alteração do esquema corporal são inconvenientes a considerar na utilização prolongada.

Assim, quando a indicação para nutrição entérica tem uma duração superior a 4-8 semanas deverá optar-se pela gastrostomia. ${ }^{1,2} \mathrm{O}$ principal inconveniente das gastrostomias é inerente à invasividade do procedimento.

\section{Gastrostomias}

Cirúrgicas: A sua realização tem sido limitada aos doentes que têm uma indicação concomitante para laparotomia/laparoscopia (p.e. cirurgia anti-refluxo) e, com carácter de antecipação, no decurso de uma laparotomia em que é previsível a necessidade de descompressão eficaz e/ou nutrição entérica no pós-operatório. Poderá ainda ter que se realizar uma gastrostomia cirúrgica se existir contraindicação para gastrostomia (endoscópica) percutânea, como seja a incapacidade de insuflar o estômago e a estenose esofágica grave. As gastrostomias clássicas (p.e. Stamm) apresentam inconvenientes relacionados com a colocação e fixação da sonda/tubo, pelo que têm sido preteridas em favor da colocação dos denominados botões de gastrostomia (Fig. 1), com vantagens psicológicas e de independência para o doente. Estes dispositivos permitem a conexão de sondas de administração e de descompressão e possuem um mecanismo valvular anti-refluxo. Além das complicações inerentes à existência da gastrostomia (extravasamento, infeção local, etc.), os botões podem apresentar complicações relacionadas com o próprio dispositivo tais como incontinência da válvula, exteriorização e deterioração das sondas de conexão. A durabilidade média é de cerca de seis meses mas pode prolongar-se até aos dois anos.

A cirurgia de acesso minimamente invasivo (laparoscópica), como procedimento isolado para a colocação de dispositivos de gastrostomia, tem vindo a demonstrar excelentes resultados, desafiando actualmente a primazia às técnicas percutâneas (ver adiante). ${ }^{14}$

Percutâneas: A colocação percutânea de dispositivos de gastrostomia (um tubo ou inclusive um botão) pode ser efetuada sob controlo radiológico ou sob controlo endoscópico (eventualmente assistida por laparoscopia). Existem diversos métodos para colocação endoscópica, sendo a técnica de pull (tração) a mais utilizada (Fig. 2); esta foi executada pela primeira vez em 1979, num lactente, no Children's Hospital da Universidade de Cleveland, por Michael Gauderer e Jeffrey Ponsky, o primeiro dos quais cirurgião pediátrico e considerado o mentor da técnica. ${ }^{15,16}$

A gastrostomia percutânea, nomeadamente a endoscópica (PEG), apresenta diversas vantagens relativamente às gastrostomias cirúrgicas 'clássicas' tais como: ausência de cicatriz, menos aderências intra-abdominais, menor tempo



Figura 1 - Botão de gastrostomia (Mic-Key, Kimberley-Clark. Ballard Medical Products) 

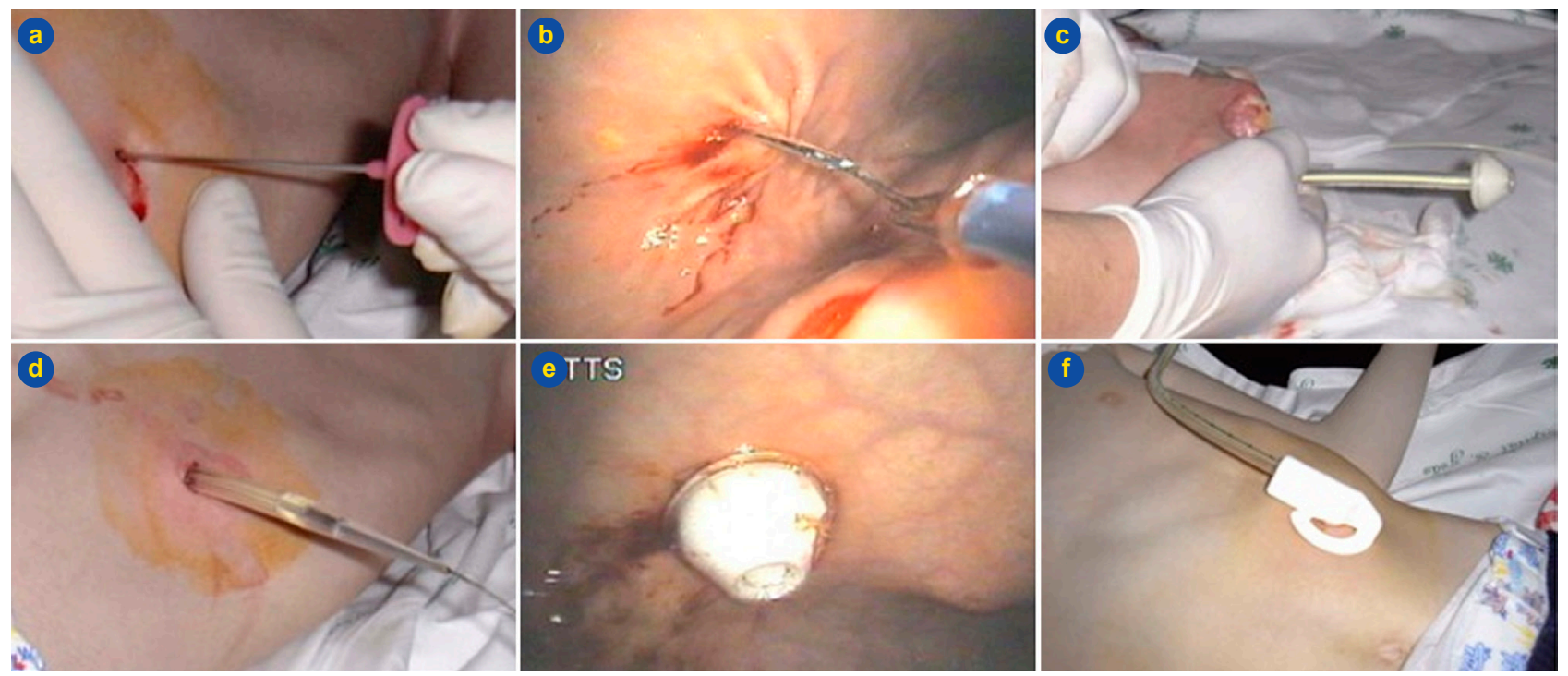

Figura 2 - Colocação de gastrostomia endoscópica percutânea (PEG). Após identificação, por transiluminação da parede abdominal, é efetuada a punção gástrica transparietal (a); após passagem de fio-guia e sua recuperação intragástrica (b) é realizado o seu transporte retrógrado até ao exterior; após ancoragem do fio-guia à extremidade distal do tubo de gastrostomia é efetuada a sua passagem anterógrada (pull) e exteriorização pela parede abdominal $(\mathrm{c}, \mathrm{d})$; por fim, procede-se à verificação da coaptação da extremidade proximal do tubo (e) e à fixação/adaptação do tubo de gastrostomia (f).

operatório, menos dor, menor tempo de hospitalização e menor custo financeiro. Além disso, pode ser utilizada precocemente, geralmente após 6-24 horas, ${ }^{2}$ embora seja considerada segura a administração praticamente imediata ${ }^{17}$; a ocorrência de extravasamento também é significativamente menor que nas gastrostomias cirúrgicas 'clássicas'. ${ }^{18}$ Contudo, estas vantagens esbatem-se face à colocação cirúrgica por laparoscopia, nomeadamente por ser viável realizar a gastrostomia, com colocação imediata de botão, sem cicatriz adicional (Fig. 3); acresce que esta técnica, por disponibilizar visualização direta de todo o procedimento, permite eleger com maior acuidade o local da gastrostomia e apresenta uma incidência significativamente menor de complicações graves. ${ }^{19}$ Contudo, está por estabelecer qual das técnicas (PEG vs laparoscopia) apresenta o binómio risco-benefício mais vantajoso.

Para a colocação de uma PEG não é imprescindível a anestesia geral em bloco operatório, podendo ser efetuada com segurança sob sedação e anestesia local, inclusivamente na criança. A taxa de êxito na colocação é de cerca de $90 \% .^{2}$

As complicações da PEG são relativamente frequentes, podendo atingir taxas de $8-30 \%$; em séries com seguimento longo, a taxa de complicações pode mesmo chegar aos $73 \% .^{2,20,21}$ As complicações major (peritonite, hemorragia, lesão visceral) são, contudo, excecionais mas não desprezíveis. ${ }^{20-25} \mathrm{~A}$ incidência de infeção local do ostoma pode ser significativamente diminuída pela antibioprofilaxia. ${ }^{2} \mathrm{~A}$ exteriorização da sonda é geralmente devida a excesso de tensão na fixação externa. A separação do estômago da parede ocorre geralmente durante a substituição por outra sonda/tubo ou por botão; é aconselhável só efetuar a primeira substituição pelo menos oito semanas após a colocação, podendo este intervalo chegar aos seis meses para crianças malnutridas e/ou em corticoterapia. As fístulas gastroentéricas ocorrem em aproximadamente 2-3\% dos casos; ${ }^{26}$ de forma a evitar a fístula gastrocólica, a insuflação gástrica com perfeita identificação do local da punção gástrica (através da transiluminação cutânea e da visualização endoscópica da indentação por pressão digital da parede abdominal) é um requisito 'quase' obrigatório. Existem ainda complicações relativamente raras tais como a laceração do esófago e a oclusão intestinal. A colocação de uma PEG requer assim um gastroenterologista experiente e uma unidade diferenciada com o apoio de um cirurgião pediátrico, pois as complicações são frequentemente cirúrgicas. ${ }^{24}$

Embora existam relatos de séries com resultados discordantes, a colocação de gastrostomia sob controlo radiológico apresenta, em geral, uma taxa de complicações mais alta sobretudo das consideradas graves, que a registada para a PEG. ${ }^{23,27-29}$

A colocação de uma PEG assistida por laparoscopia é uma alternativa que se tem revelado eficaz na prevenção de complicações graves, nomeadamente minimizando/anulando o risco de lesão de órgãos adjacentes e permitindo uma melhor escolha da localização da gastrostomia. ${ }^{24,25,30-34}$

Cerca de $3 / 4$ dos orifícios de gastrostomia encerram espontaneamente após a remoção da sonda/botão. A obliteração é, por vezes, tão rápida que no caso de exteriorização acidental se deve recomendar a reintrodução imediata ou, caso tal não seja exequível, a introdução de uma sonda de Foley.

Dada a raridade de complicações graves, a PEG é considerada uma técnica eficaz e segura para se estabelecer um acesso de nutrição entérica mesmo em crianças com doença grave, pois os problemas são geralmente minor e relacionados com o ostoma; a grande maioria dos pais e cuidadores atribui uma influência positiva à PEG. ${ }^{2,20}$ 

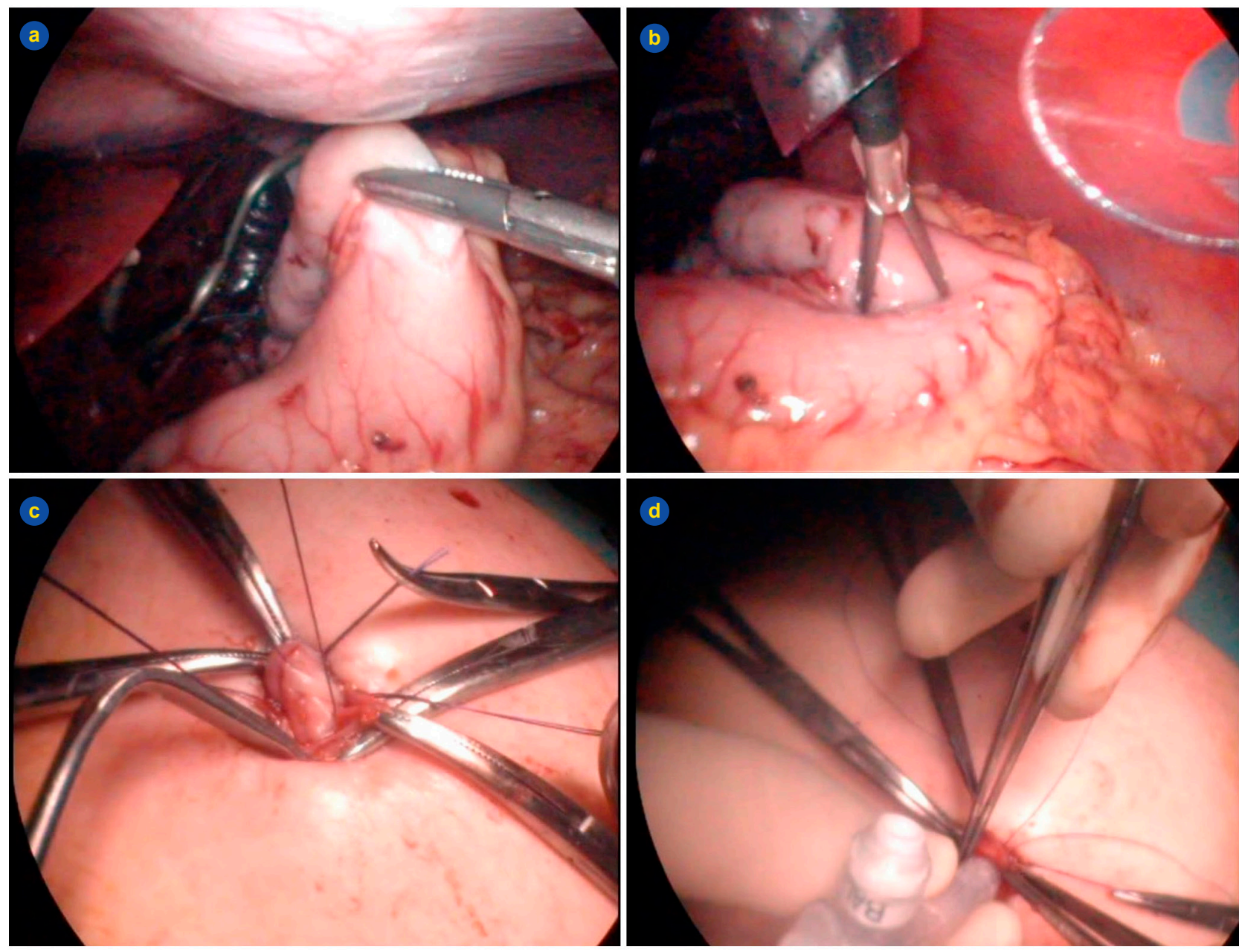

Figura 3 - Realização de gastrostomia laparoscópica. Após introdução de telescópio trans-umbilical (5 mm) e identificação do estômago, elege-se a localização da gastrostomia por uma de duas formas: colocação de um acesso de 3-5 mm e preensão do estômago 'simulando' a sua exteriorização (a) ou efetuando indentação da parede abdominal por pressão digital; de seguida coloca-se um acesso de $10 \mathrm{~mm}$ na localização eleita, através do qual se faz a preensão (b) e exteriorização da parede gástrica conjuntamente com a retirada do trócar (c); após sutura do plano músculo-aponevrótico à serosa gástrica (opcional), realiza-se a gastrotomia e colocação/fixação (por balão) do botão de gastrostomia (d).

Doença do Sistema Nervoso Central: Neste grupo de crianças é frequente a indicação para colocação de gastrostomia, por motivo nutricional, devido a dificuldade da alimentação oral por incoordenação faríngea e/ou dismotilidade esofágica.

No entanto, $15-75 \%$ destes doentes apresentam RGE patológico de difícil controlo com tratamento médico. ${ }^{35}$ Para obviar o efeito nefasto de uma gastrostomia na presença de RGE, este deverá ser previamente rastreado. Perante RGE patológico deverá ser ponderada cirurgia anti-refluxo concomitante pois, apesar de em alguns doentes poder ocorrer melhoria, o RGE tende a persistir ou a agravar. ${ }^{36}$ $\mathrm{Na}$ ausência de RGE não deverá realizar-se cirurgia anti-refluxo, pois a PEG de per se não parece estar relacionada com a ocorrência subsequente de RGE ${ }^{36,37}$ Contudo, deve ser tido em conta que uma proporção substancial destas crianças vem a desenvolver RGE patológico em consequência da progressão da doença neurológica; na realidade, a doença do SNC é o único factor de risco associado à necessidade de cirurgia anti-refluxo após colocação de

\section{PEG. ${ }^{38}$}

\section{Sonda Naso(duodenal)jejunal e Jejunostomias}

Como referido anteriormente, a infusão pós-pilórica constitui uma segunda linha para nutrição entérica, nomeadamente quando a administração gástrica não é tolerada e/ ou existe alto risco de aspiração traqueobrônquica.

A colocação 'às cegas' de uma sonda naso(duodenal) jejunal (Fig. 4), com ou sem medidas adicionais tais como a insuflação gástrica ou a administração de eritromicina, pode ter uma taxa de êxito até $75-90 \%$, sobretudo quando existem equipas e programas de treino específicos; ${ }^{39-41}$ aquela taxa é sobreponível à verificada para a colocação sob controlo radiológico ou endoscópico. ${ }^{42,43}$

Perante indicação para alimentação entérica pós-pilórica de longa duração (superior a um mês), deve ser ponderada a colocação de jejunostomia. A colocação percutânea indireta, através de uma gastrostomia, é uma técnica viável, mas a alta taxa de problemas relacionada com os tubos gastrojejunais (obstrução, deslocação, etc.) obsta a 


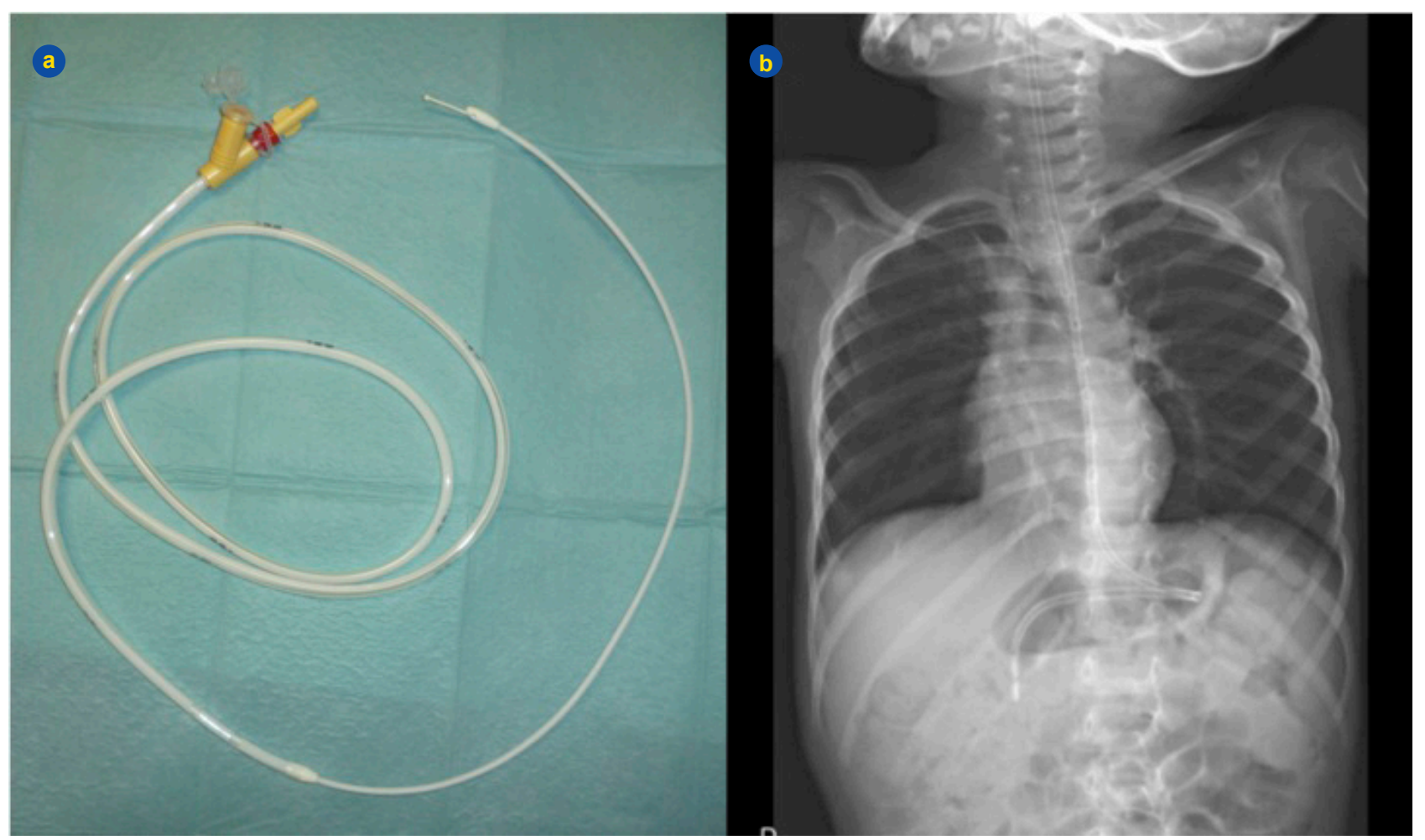

Figura 4 - a) Sonda nasojejunal (Compat Stay put 9/18Fr, Nestlé HealthCare Nutrition GmbH); b) Sonda nasoduodenal (Rx).

que seja considerada, atualmente, como uma alternativa eficaz para alimentação entérica de longo prazo.44-46 No que respeita à colocação de uma jejunostomia percutânea sob controlo endoscópico, a experiência atual em crianças é muito limitada. ${ }^{47,48} \mathrm{~A}$ realização de uma jejunostomia cirúrgica, nomeadamente se assistida por laparoscopia, é uma técnica eficaz e minimamente invasiva que deve assim ser considerada precocemente. ${ }^{49}$

Por último, deve ser tido em linha de conta que os acessos pós-pilóricos podem apresentar praticamente todas as complicações equivalentes às das gastrostomias, a que acresce uma incidência significativamente maior de distúrbios gastrointestinais específicos (p.e. jejunoileíte, invaginação, vólvulo intestinal $)^{47,50,51}$ e uma taxa de mortalidade mais alta. ${ }^{2}$

\section{CONCLUSÃO}

A nutrição entérica deverá ser efetuada, preferencialmente, através da administração intragástrica. A infusão através de sonda nasogástrica constitui uma modalidade simples, mas de utilização limitada em termos de duração; quando a indicação se mantém para além dos dois meses

\section{REFERÊNCIAS}

1. Teitelbaum DH, Coran AG. Nutritional support. In: Grosfeld JL, O'Neill Jr JA, Fonkalsrud EW, editors. Pediatric Surgery. $6^{\text {th }}$ ed. Philadelphia: Mosby Elsevier; 2006. p.194-220.

2. Braegger C, Decsi T, Dias JA, Hartman C, Kolacek S, Koletzko B, et al. Practical approach to pediatric enteral nutrition: a comment by the ESPGHAN committee on nutrition. J Pediatr Gastroenterol Nutr. 2010;51:110-22.

3. Ohta K, Omura K, Hirano K, Kanehira E, Ishikawa N, Kato Y, et al. The effects of small amounts of a low residual diet against total parenteral deverá ponderar-se a colocação de uma gastrostomia. A gastrostomia endoscópica percutânea é um procedimento eficaz e seguro, embora com morbilidade minor frequente e não isenta de complicações graves; a colocação de uma gastrostomia cirúrgica por laparoscopia é uma alternativa potencialmente vantajosa por permitir a visualização de todo o procedimento. Quando a utilização do estômago é inviável ou comporta alto risco de aspiração, está indicada a administração pós-pilórica. A infusão através de uma sonda naso(duodenal)jejunal é uma opção eficaz, embora a colocação e manutenção requeira uma equipa treinada e experiente; quando a indicação para nutrição jejunal é prolongada, as alternativas à jejunostomia cirúrgica ainda não se encontram devidamente validadas na criança.

\section{CONFLITOS DE INTERESSE}

O autor declara que não houve conflitos de interesse na realização deste trabalho.

\section{FONTES DE FINANCIAMENTO}

O autor declara não terem existido fontes de financiamento externas na realização deste trabalho. nutrition. Am J Surg. 2003;185:79-85

4. Tyson JI, Kennedy KA. Minimal enteral nutrition for promoting tolerance and preventing morbidity in parenterally fed infants. Cochrane Database Syst Rev. 2005;3:CD000504

5. Dwyer KM, Watts DD, Thurber JS, Benoit RS, Fakhry SM. Percutaneous endoscopic gastrostomy: the preferred method of elective feeding tube placement in trauma patients. J Trauma. 2002;52:26-32.

6. Kreis BE, Middelkroop E, Vloemans AF, Kreis RW. The use of PEG tube in a burn centre. Burns. 2002;28:191-7. 
7. Sefton EJ, Boulton-Jones JR, Anderton D, Teahon K, Knights DT. Enteral feeding in patients with major burn injury: the use of nasojejunal feeding after failure of nasogastric feeding. Burns. 2002;28:386-90.

8. Stanga Z, Giger U, Marx A, Delegge MH. Effect of jejunal long-term feeding in chronic pancreatitis. J Parenter Enteral Nutr. 2005;29:12-20.

9. Van Biervliet S, De Waele K, Van Winckel M, Robberecht E. Percutaneous endoscopic gastrostomy in cystic fibrosis: patient acceptance and effect of overnight tube feeding on nutritional status. Acta Gastroenterol Bel. 2004;67:241-4.

10. Metheny N. Measures to test placement of nasogastric and nasointestinal feeding tubes: a review. Nurs Res. 1988;37:324-9.

11. Puntis JW. Nutritional support at home and in the community. Arch Dis Child. 2001;84:295-8.

12. Mittal RK, McCallum RW. Characteristics of transient lower esophageal sphincter relaxation in humans. Am J Physiol. 1987;G636-6.

13. Cucchiara S, Birtolotti M, Minella R, Auricchio S. Fasting and postprandial mechanisms of gastroesophageal reflux in children with gastroesophageal reflux disease. Dig Dis Sci. 1993;38:86-92.

14. Jones VS, La Hei ER, Shun A. Laparoscopic gastrostomy: the preferred method of gastrostomy in children. Pediatr Surg Int. 2007;23:1085-9.

15. Gauderer MW, Ponsky JL, Izant RJ. Gastrostomy without laparotomy: a percutaneous endoscopic technique. J Pediatr Surg. 1980;15:872-5.

16. Gauderer MW. Percutaneous endoscopic gastrostomy- 20 year later: a historical perspective. J Pediatr Surg. 2001;36:217-9.

17. Corkins MR, Fitzgerald JF, Gupta SK. Feeding after percutaneous endoscopic gastrostomy in children: early feeding trial. J Pediatr Surg. 2010;50:625-7.

18. Turial S, Schwind M, Engel V, Kohl M, Goldinger B, Schier $F$ Microlaparoscopic-assisted gastrostomy in children: early experiences with our technique. J Laparoendosc Adv Surg. 2009;19:S229-31.

19. Akay B, Capizzani TR, Lee AM, Drongowski RA, Geiger JD, Hirschl RB, et al. Gastrostomy tube placement in infants and children: is there a preferred technique? J Pediatr Surg. 2010;45:1147-52.

20. Avitsland TL, Kristensen C, Emblem R, Veenstra M, Mala T, Bjornland K. Percutaneous endoscopic gastrostomy in children: a safe technique with major symptom relief and high parental satisfaction. J Pediatr Gastroenterol Nutr. 2006;43:624-8.

21. Ségal D, Michaud L, Guimber D, Ganga-Zandzou PS, Turck D, Gottrand F. Late-onset complications of percuateneous endoscopic gastrostomy in children. J Pediatr Gastroenterol Nutr. 2001;33:495-500.

22. Beres A, Bratu I, Laberge JM. Attention to small details: big deal for gastrostomies. Semin Pediatr Surg. 2009;18:87-92.

23. Nah SA, Narayanaswamy B, Eaton S, Coppi PD, Kiely EM, Curry JI, et al. Gastrostomy insertion in children: percutaneous endoscopic or percutaneous image-guided? J Pediatr Surg. 2010;45:1153-8.

24. Vervloessem D, van Leersum F, Boer D, Hop WC, Escher JC, Madern $\mathrm{GC}$, et al. Percutaneous endoscopic gastrostomy (PEG) in children is not a minor procedure: risk factors for major complications. Semin Pediatr Surg. 2009;18:93-7.

25. Zamakhshary M, Jamal M, Blair GK, Murphy JJ, Webber EM, Skarsgard ED. Laparoscopic vs percutaneous endoscopic gastrostomy tube insertion: a new pediatric gold standard? J Pediatr Surg. 2005;40:85962.

26. Patwardham N, McHugh K, Drake D, Spitz L. Gastroenteric fistula complicating percutaneous endoscopic gastrostomy. J Pediatr Surg. 2004;39:561-4.

27. Akinci C, Ciftci TT, Kaya D, Ozmen MN, Akhan O. Long-term results of percutaneous radiologic gastrostomy and gastrojejunostomy in children with emphasis on technique: single or double gastropexy? Am J Roentgenol. 2010;195:1231-7.

28. Friedman JN, Ahmed S, Connolly B, Chait P, Mahant S. Complications associated with image-guided gastrostomy and gastrojejunostomy tubes in children. Pediatrics. 2004;114:458-61.

29. Lewis EC, Connolly B, Temple M, John P, Chait PG, Vaughan J, et al. Growth outcomes and complications after radiologic gastrostomy in 120 children. Pediatr Radiol. 2008;38:963-70.
30. Charlesworth P, Hallows M, van der Avoirt A. Single-center experience of laparoscopically assisted percutaneous endoscopic placement. J Laparoendosc Adv Surg Tech A. 2010;20:73-5.

31. Idowu O, Driggs XA, Kim S. Laparoscopically assisted antegrade percutaneous endoscopic gastrostomy. J Pediatr Surg. 2010;45:277-9.

32. Lotan G, Broide E, Efrati Y, Klin B. Laparoscopically monitored percutaneous endoscopic gastrostomy (PEG) in children: a safer procedure. Surg Endosc. 2004;18:1280-2.

33. Smitherman S, Pimpalwar A. Laparoendoscopic gastrostomy tube placement: our all-in-one technique. J Laparoendosc Adv Surg Tech A 2009;19:119-23.

34. Takahashi T, Okazaki T, Kato Y, Watayo H, Lane GJ, Kobayashi H, et al Laparoscopy-assisted percutaneous endoscopic gastrostomy. Asian J Surg. 2008;31:204-6.

35. Gustafsson PM, Tibbling L. Gastro-esophageal reflux and oesophagea dysfunction in children and adolescentes with brain damage. Acta Paediatr. 1994;83:1081-5.

36. Samuel M, Holmes K. Quantitative and qualitative analysis of gastroesophageal reflux after percutaneous endoscopic gastrostomy. J Pediatr Surg. 2002;37:256-61.

37. Razeghi S, Lang T, Behrens R. Influence of percutaneous endoscopic gastrostomy on gastroesophageal reflux: a prospective study in 68 children. J Pediatr Gastroenterol Nutr. 2002;35:27-30.

38. Novotny NM, Jester AL, Ladd AP. Preoperative prediction of need for fundoplication before gastrostomy tube placement in children. J Pediatr Surg. 2009;44:173-6.

39. Meyer R, Harrison S, Cooper M, Habibi P. Successful blind placement of nasojejunal tubes in paediatric intensive care: impact of training and audit. J Adv Nurs. 2007;60:402-8.

40. Phipps LM; Weber MD, Ginder BR, Hulse MA, Thomas NJ. A randomized controlled trial comparing three different techniques of nasojejunal feeding tube placement in critically ill children. J Parenter Enteral Nutr. 2005;29:420-4.

41. Levy H, Hayes J, Boivin M, Tomba T. Transpyloric feeding tube placement in critically ill patients using electromyogram and erythromycin infusion. Chest. 2004;125:587-91.

42. Vitta L, Raghavan A, Morrell R, Sprigg A. Fluoroscopy-guided insertion of nasojejunal tubes in children- setting local diagnostic reference levels. Pediatr Radiol. 2009;39:1203-8.

43. Zhihui T, Wenkui $Y$, Weiqin L, Zhiming W, Xianghong $Y$, Ning L, et al. A randomized clinical trial of transnasal endoscopic versus fluoroscopy for the placement of nasojejunal feeding tubes in patients with severe pancreatitis. Postgrad Med J. 2009;85:59-63.

44. Doede T, Faiss S, Schier F. Jejunal feeding tubes via gastrostomy in children. Endoscopy. 2002;34:539-42

45. Fortunato JE, Darbari A, Mitchell SE, Thompson RE, Cuffari C. The limitations of gastro-jejunal (G-J) feeding tubes in children: a 9-year pediatric hospital database analysis. Am J Gastroenterol. 2005;100:1869.

46. Godbole P, Margabanthu G, Crabbe DC, Thomas A, Puntis JW, Abe $\mathrm{G}$, et al. Limitations and uses of gastrojejunal feeding tubes. Arch Dis Child. 2002;86:134-7.

47. Freeman C, Delegge MH. Small bowel endoscopic enteral access. Curr Opin Gastroenterol. 2009;25:155-9.

48. Virnig DJ, Frech EJ, Delegge MH, Fang JC. Direct percutaneous endoscopic jejunostomy: a case series in pediatric patients. Gastrointest Endosc. 2008;67:984-7

49. Esposito C, Settimi A, Centonze A, Capano G, Ascione G Laparoscopic-assisted jejunostomy: na effective procedure for the treatment of neurologically impaired children with feeding problems and gastroesophageal reflux. Surg Endosc. 2005;19:501-4.

50. Garrett-Cox R, Richards CA, Misra D. Severe jejunoileitis after placement of a feeding jejunostomy: a series of four cases and a review of the literature. J Pediatr Surg. 2003;38:1090-3.

51. Hughes U, Connolly B. Small-bowel intussusceptions occurring around nasojejunal enteral tubes- three cases occurring in children. Pediatr Radiol. 2001;31:456-7. 


\section{Vias para Nutrição Artificial na Criança (I): Acessos Entéricos}

Acta Med Port 2014:27:634-640

Publicado pela Acta Médica Portuguesa, a Revista Científica da Ordem dos Médicos

Av. Almirante Gago Coutinho, 151

1749-084 Lisboa, Portugal.

Tel: +351218428215

E-mail: submissao@actamedicaportuguesa.com

www.actamedicaportuguesa.com

ISSN:0870-399X | e-ISSN: 1646-0758

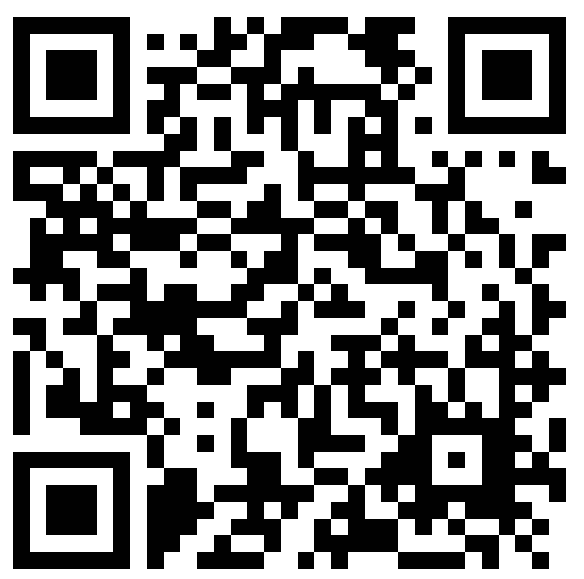

